\title{
Designing Physical Human-Machine-Interfaces for Exoskeletons Using 3D-Shape Analysis
}

\author{
Christine LINNENBERG ${ }^{1}$, Robert WEIDNER ${ }^{1,2}$ \\ ${ }^{1}$ Leopold-Franzens-Universität Innsbruck, Innsbruck, Austria; \\ ${ }^{2}$ Helmut Schmidt University/University of the Federal Armed Forces Hamburg, Hamburg, Germany
}

https://doi.org/10.15221/19.085

\begin{abstract}
Exoskeletons are a promising future technology to assist workers in high demanding workplaces (e.g., activities in or above head level or lifting heavy loads) and to reduce critically strained body parts (e.g., shoulder or back) [1]. Classical exoskeletons generally consist of a mechanical structure parallel to the body segments, actuators for force generation, a control unit (in case of active systems) and human-machine-interfaces (HMI). HMI either fix the exoskeleton to the body or transfer supporting forces from the exoskeleton to the human endoskeleton. At the same time, they need to satisfy safety and comfort requirements [2]. Concerning force transfer too high pressure as well as play within the HMI must be avoided. Precise knowledge of the anthropometric changes in various body positions and muscle states is, therefore, the prerequisite for the design and dimensioning of HMI.

This article presents a procedure for designing individual HMI based on 3D shape analysis using the example of activities at head level or above. Based on the general approach [3] the evaluation of the anthropometric changes of upper extremities due to different limb positions and muscle states (relaxed and tensed) is described. Furthermore, the process of data fitting for integrating the individual body parameters into the CAD-construction process is introduced.

Three human subjects were integrated into the experimental study, representing different figure types according to ISO 8559-2:2017 [4]. Body postures were scanned using the 3D laser body scanner VITUSbodyscan (Vitronic $\mathrm{GmbH}$ ), characterizing daily postures of production activities in or above head level as an example for industrial work. In order to compare the scans in relaxed and tensed muscle states as well as in different limb positions with high precision, physical markers and handholds were used. Raw scans were processed and reconstructed with ArthroScan Software tools (Avalution $\mathrm{GmbH}$ ), 3D shape analysis was calculated for anthropometric changes of the upper arm. In a first step, orthogonal planes to the upper arm were generated and multiplied using Geomagic Freeform to gain intersections with the arm. In the next step, the intersections were fitted into ellipses using Matlab. Finally, the ellipse data formed the basis for the automated CAD-process to create an individual HMI. The results of the study reveal a strong indication that individual human-machine-interfaces are needed to achieve satisfaction concerning fitting, force transfer and safety aspects.
\end{abstract}

Keywords: 3D body scanning, anthropometrics, exoskeletons, human-machine-interface, design process

\section{Background}

The development of exoskeletons already began in the $1960^{\text {s }}$ with the invention of the mobile Hardiman that was supposed to augment human strength and endurance [5]. With the growing technical opportunities, exoskeletons became lighter and faster. Their original applications are mainly in the medical and rehabilitation sector as well as in military and civilian service. Despite a broad automation, manual work is still not to be missed in manufacturing production, assembly and logistics. Also in these fields, exoskeleton systems are a promising technology to assist people in high demanding tasks (e.g., lifting heavy loads or working in or above head level). It was shown that several systems reduce the physical [6] and cognitive load [7] for specific work tasks and enhance productivity and quality of life.

Classical exoskeletons consist of rigid structures parallel to the body segments, control units (in case of active devices), actuators (e.g., electric engines, pneumatic actuators or springs) and human-machine-interfaces.

Primarily human-machine-interfaces have to fulfill two requirements: The first one is to fix the exoskeleton to the body, the second requirement is to transfer the forces generated by the actuators to the endoskeleton and support the user in his movements. The effectivity of an exoskeleton depends thereby amongst others on the human-machine-interfaces [8]. If the human-machine-interface is not transferring the forces in an ideal manner, it is not just uncomfortable after prolonged usage [2] but leads to frequent skin and soft tissue damages [9]. The deformation of the soft tissue causes a delay in 
the support effect and thus leads to incongruences of the joint axis and asynchronous motion sequences with higher neuromuscular and structural strains and increased metabolic rates [10].

In most exoskeleton systems, human-machine-interfaces for the extremities are designed as hard shells with circular tightening straps.

From a technical point of view, soft tissue is considered as a confounding variable in the coupling of technical systems to humans due to their high deformability. For a direct, unimpeded power transmission hard, superficial bone structures would be suitable. However, these structures are usually located at the joints itself, which offer only poor leverage. Considering the multiple degrees of freedom, superficial bone structures are given only in small quantity and quality. Therefore, there is a high necessity to consider soft tissue and its properties in the physical coupling of humans and technology. During power transmission, deformation is unavoidable, but in particular, vascular and neuronal structures within the soft tissues must be protected against excessive punctual pressure forces and shear forces resulting from play within the interface itself. Exact knowledge of the anthropometric characteristics at the intended interface is therefore incessant.

\section{State of the art}

In the following, the development of human-machine-interfaces within the field of orthopedic technology will be described. In order to deduce the need for action for human-machine-interfaces for exoskeletons the current methods for acquiring anthropometric characteristics will be presented as well.

\subsection{Development of orthoses and orthotic support systems}

Know-how in the field of human-machine-interfaces is provided by the field of orthopedic technology, especially by orthotics and prosthetics. Fabric-based orthoses are usually ready-made products whose stabilization effect is achieved less by mechanical properties of the orthosis but rather by a sensory component [11]. They are generally based on 1D data from anthropometric series measurements and sometimes require individual, professional adaptation to the user. Prostheses or prosthetic stems, on the other hand, are custom-made, highly individual and cost-intensive. The current practice of manufacturing by negative-positive casting with plaster is slowly replaced by CAD methods based on individual 3D-body scanning. For some years, however, 3D-data has also been increasingly used in the development of soft orthoses, for example, in the EU project FASHION-ABLE [12] or lumbar orthotics for obese persons [13].

\subsection{Anthropometry, virtual body models and fit design}

Serial measurements of anthropometric data using 3D full-body scanning techniques were conducted with men, women and children (SizeGermany) for four basic positions (a) upright seat, (b) stance with relaxed arms (c) wide stance with arms slightly abducted, (d) wide stance with arms stretched forward [14]. Equivalent European and American data were collected in various projects (including Sizeltaly, CNM, SizeNorthAmerika) [15]. On this basis, representative avatars can be calculated for different body sizes and typical shapes (from very slim to very strong). For example, in the garment industry, 1D body measurements (lengths and perimeters of body segments) are extracted by default and used for the development of CAD sewing patterns [14]. Lines are then checked on fitting errors and pattern are readjusted with the aid of the resulting avatars from the serial measurements [16]. The mentioned tools and the results of the serial measurements also incorporate product engineering (e.g., automotive and aircraft industry [17]) or serve as the basis for the production of made-to-measure skiing boots.

Especially in workwear and personal protective equipment (PPE) optimal freedom of movement in addition to protection and function is central for users and developers. The first 3D anthropometry measurements were taken by the Institut Hohenstein. Those measurements took, in addition to the basic positions, six further postures such as forward bending, squat, or sitting with raised arms into account [18].

However, the accepted tolerances for workwear or personal protective equipment are very different from those for human-machine-interfaces for power transmission [3]. Thus, it is not recommended to make use of the existing 3D anthropometric measurements for human-machine-interfaces of exoskeletons. Instead, anthropometry measurements tailored to the specific body part should be conducted for the development of those specific systems.

Therefore, this paper presents a method for detecting deformation of specific body segments via 3D laser body scanning based on the example of the upper arm. Furthermore, the paper points out, how the shape and deformation can be transformed into simple geometric patterns in order to use them for the development of individual interfaces. 


\section{Methods}

\subsection{Scanning procedure}

All full-body scans of this work were taken using the 3D laser body scanner VITUSbodyscan (Vitronic $\mathrm{GmbH}$ ). A scan wizard was prepared for all postures taken. A separate screen within the scanning booth informed the study participant about the actual posture by picture and description. Despite the area of interest, all participants wore short tights and a scanning cap. For later analytics, a physical marker was placed two centimeters below deltoid tuberosity of the humerus, where skin shift over different arm positions is minimal. Handholds were installed within the booth and adjusted to the participants' height to avoid motion artifacts.

In order to design an human-machine-interface for the upper arm for an exoskeleton that supports manual activities in or above head level, different dynamic postures representing daily dynamic postures of manufacturing task (e.g., overhead drilling, car assembly or varnishing) were taken. Considering the degrees of freedom from shoulder and elbow and the natural free scope of working in or above head level, a total number of eleven dynamic postures were scanned. With regard to the deformation of the soft tissue in relaxed and tense conditions, all eleven postures were scanned in both muscle states. To avoid occultation, only the right arm adopted the specific working posture, whereas the left arm was kept in standard position.

For the measurements, eleven postures were necessary, which are summarized in figure 1: (1) standard posture, (2) standard posture with straight arm, (3) straight arm, 120 flexed shoulder, (4) $\sim 30^{\circ}$ flexed elbow with supination, $\sim 70^{\circ}$ flexed shoulder, (5) $30^{\circ}$ flexed elbow with pronation, $\sim 70^{\circ}$ flexed shoulder, (6) $\sim 90^{\circ}$ flexed elbow with supination, $\sim 70^{\circ}$ flexed shoulder, (7) $\sim 90^{\circ}$ flexed elbow with pronation, $\sim 70^{\circ}$ flexed shoulder, (8) $\sim 90^{\circ}$ flexed elbow with supination, $\sim 120^{\circ}$ flexed shoulder, (7) $\sim 90^{\circ}$ flexed elbow with pronation, $\sim 120^{\circ}$ flexed shoulder, (10) $\sim 90^{\circ}$ flexed elbow, $\sim 70^{\circ}$ flexed shoulder with inner rotation, (11) $\sim 90^{\circ}$ flexed elbow, $\sim 120^{\circ}$ flexed shoulder with inner rotation.

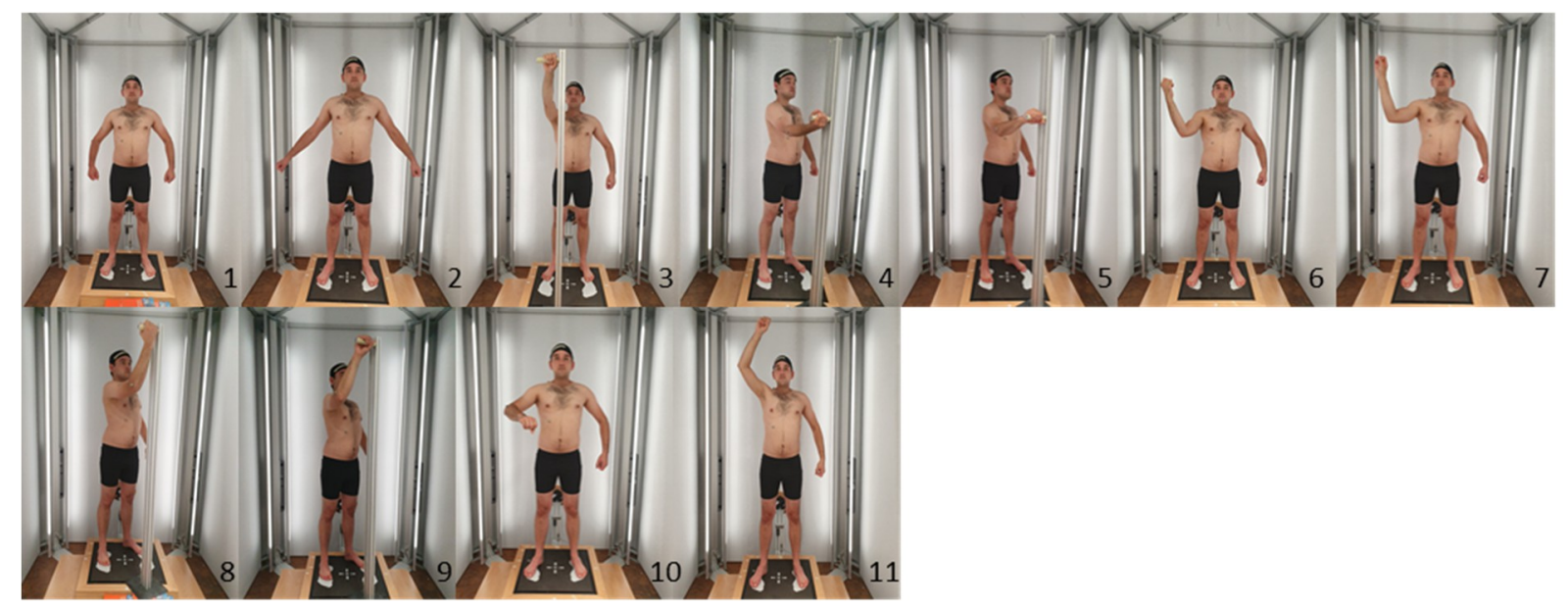

Fig. 1: Postures scanned representing daily postures in manufacturing

\subsection{Post-processing and statistical analysis}

In the second part of the study, the physical markers were marked manually, and their coordinates were exported separately. All scans were reconstructed in arbitrary geometries mode except the standard posture, which was reconstructed in human/standard posture mode. All scans were exported as .obj-Files. In a next step, each .obj-Files was imported into Geomagic Freeform, which was used to construct an orthogonal plane to the arm at the height of the physical marker. The orthogonal plane was multiplied nine times in central and distal direction with a distance of $10 \mathrm{~mm}$ between each plane, compare Figure 2. The intersection between object-surface and each plane was exported as .igs-Files. 

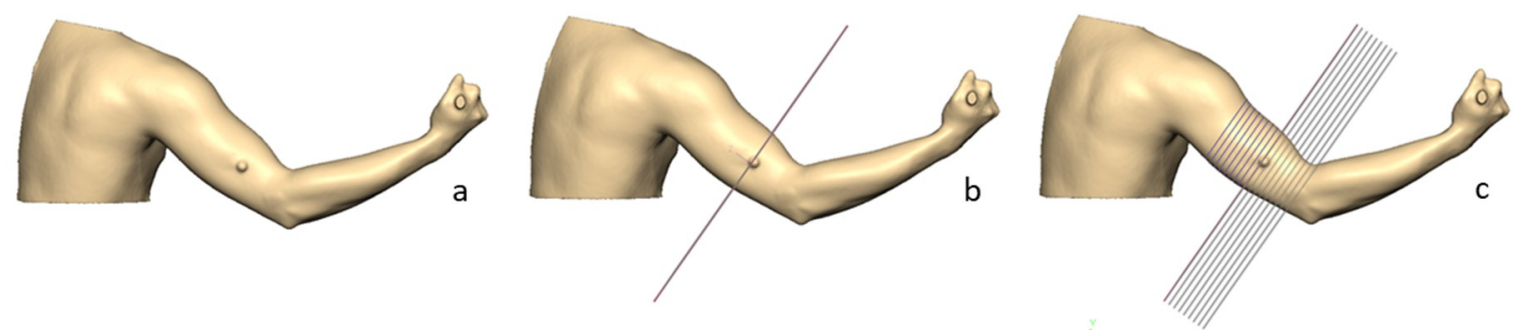

Fig. 2: Processed scan of the upper arm within Geomagic Freeform, (a) with a physical marker, (b) with an orthogonal plane and (c) with multiple planes and intersections

After this, invalid intersection curves were identified in MATLAB. If the angle of the elbow was to narrow, and the intersection curve included the lower arm or in case of closed armpits the curve included parts of the chest, the intersection curves were excluded from the analysis. See figure 3, as an example.

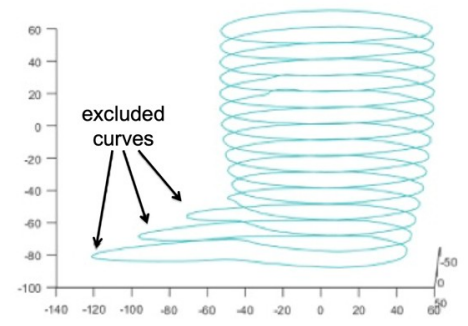

Fig. 3: Example for excluded intersection curves

Figure 4 shows the process of ellipse fitting via MATLAB. First, each intersection curve was translated and rotated to a standard position in the three-dimensional space using the midpoint of the intersection curve at the height of the physical marker.

After standardization, each intersection curve was fitted into an ellipse using the method of least squares [19]. The intersections and ellipse curve were then analyzed by circumference and length of the semi-major and semi-minor axis of the ellipse. The mean value and standard deviation over all 22 postures taken were calculated for each test subject separately to gain information on the deformation magnitude. The mean and standard deviation data were then fitted with a third order polynomial curve.

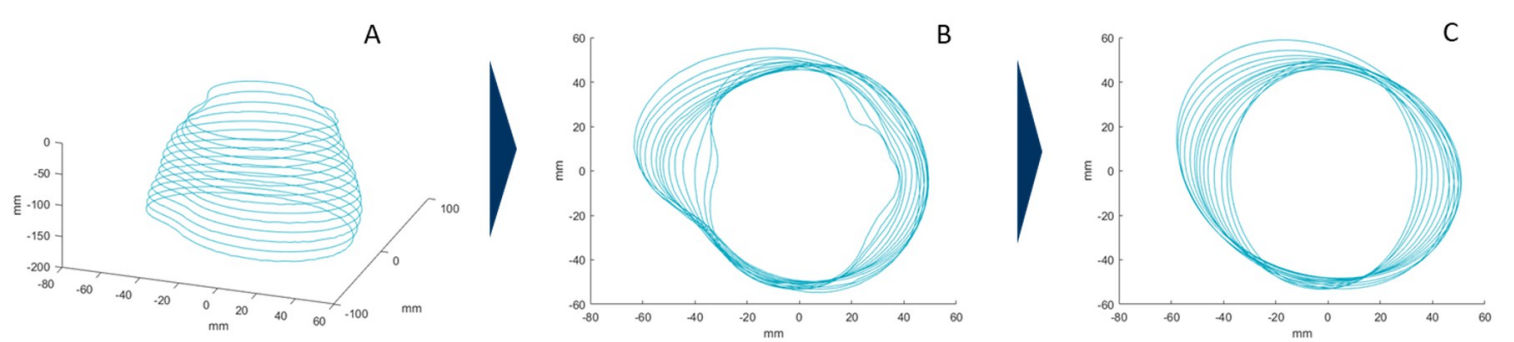

Fig. 4: Process of ellipse fitting via MATLAB, $(A)$ intersections in three dimensions, $(B)$ intersections rotated,

(C) ellipse fitting of intersections

\subsection{CAD-Process}

After the ellipse fitting the mean characteristics of the ellipses per cutting height are transferred to a parametric CAD model of the human-machine-interface.

The parametric CAD model is already predefined [3]. It includes the invariant structures of the machine-machine-linkage between the exoskeleton and the human-machine interface as well as the generalized ellipse characteristics (e.g., semi-major and semi-minor axis, centers, angles and distance between the ellipses). Ellipses are closed via spline modeling. The surface formed this way represents the inner surface of the human-machine-interface. The inner surface is then extruded and defines the preliminary human-machine-interface. A circumferential cut opens the still closed human-machine-interface. The individualization of the human-machine-interface is made automatically by changing the characteristics of the test subject-related ellipses. Figure 5 shows a predefined human-machine-interface for the upper arm. The degree of deformation of the soft tissue is considered by using corresponding resilient materials depending on the value of the standard deviation of the average shape. 


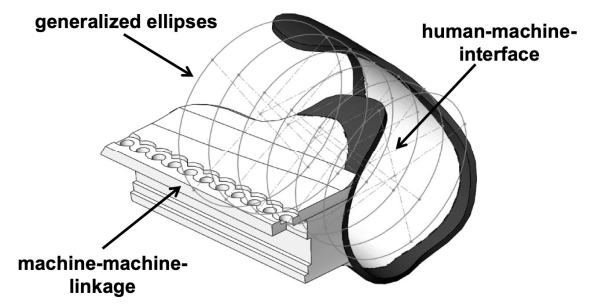

Fig. 5: CAD-model of the predefined human-machine-interface for the upper arm

\section{Results}

The results are divided in the description of the participants, results of the circumferences, and the results of the semi-major and semi-minor axis of the fitted ellipses. A further sub-division is established for the results of the circumferences into a description of the results in standard posture, the outcome of the different muscle conditions as well as resulting data over all conditions and postures taken.

\subsection{Participants}

Three male subjects were integrated into the experimental study to verify the procedure, feasibility of the study, and the usability of the emerging results for designing individual human-machine-interface. The subjects represent the three different figure types slim, normal, and extra strong according to height and chest girth [4], [20]. The average age was 31.6 years. Two subjects have an office job, while the third subject is engaged in a technical job. Subject 2 regularly exercises in his spare time. Descriptive statistics of the subjects are shown in Table 1.

Table 1: Descriptive statistics of subjects

\begin{tabular}{|c|c|c|c|}
\hline & Subject 1 & Subject 2 & Subject 3 \\
\hline Body height [cm] & 178 & 189 & 173 \\
\hline Chest girth [cm] & 99 & 113 & 121 \\
\hline Arm length [cm] & 33 & 36 & 30 \\
\hline
\end{tabular}

\subsection{Results of circumferences}

In standard posture subject 1 , and 2 show a mean increase in circumference of $0.5 \mathrm{~cm}$ per $1 \mathrm{~cm}$ height over the upper arm. Whereas subject 3 shows a more conical shape with an increase in circumference of $0.7 \mathrm{~cm}$ per $1 \mathrm{~cm}$ in height. Subject 1 has a mean arm circumference of $30.0 \mathrm{~cm}$. Although subject 2 and 3 differ a lot by body height and chest girth, their arm circumferences differ by only a few millimeters Mean circumference of the upper arm is $36.6 \mathrm{~cm}$ for subject 2 and $36.1 \mathrm{~cm}$ for subject 3 . Table 2 presents all circumferences of the three subjects by each cutting height of the upper arm.

Table 2: Circumferences in standard posture in standard posture at each cutting height [cm]

\begin{tabular}{|c|c|c|c|}
\hline Cutting height & Subject 1 & Subject 2 & Subject 3 \\
\hline $\mathbf{1}$ & 26.9 & 32.5 & 31.4 \\
\hline $\mathbf{2}$ & 27.5 & 33.2 & 31.7 \\
\hline $\mathbf{3}$ & 28.2 & 33.9 & 32.4 \\
\hline $\mathbf{4}$ & 28.8 & 34.6 & 33.3 \\
\hline $\mathbf{5}$ & 29.4 & 35.2 & 34.1 \\
\hline $\mathbf{6}$ & 29.8 & 35.6 & 34.8 \\
\hline $\mathbf{7}$ & 30.3 & 36.0 & 35.4 \\
\hline $\mathbf{8}$ & 30.6 & 36.3 & 35.9 \\
\hline $\mathbf{9}$ & 31.0 & 36.5 & 36.5 \\
\hline $\mathbf{1 0}$ & 32.2 & 37.8 & 38.0 \\
\hline $\mathbf{1 1}$ & 31.5 & 37.7 & 37.9 \\
\hline $\mathbf{1 2}$ & 31.6 & 37.8 & 38.6 \\
\hline $\mathbf{1 3}$ & 31.9 & 38.2 & 39.6 \\
\hline $\mathbf{1 4}$ & 32.3 & 38.7 & 40.4 \\
\hline $\mathbf{1 5}$ & 33.2 & 39.1 & 41.4 \\
\hline $\mathbf{1 6}$ & 33.7 & 39.6 & - \\
\hline $\mathbf{1 7}$ & 34.2 & 40.2 & - \\
\hline $\mathbf{1 8}$ & 31.1 & 37.3 & - \\
\hline $\mathbf{1 9}$ & 30.6 & 35.2 & - \\
\hline mean & 30.8 & 36.6 & 36.1 \\
\hline
\end{tabular}

Figure 6 shows a comparison of the fitted courses of the circumference of the included subjects in standard posture. 


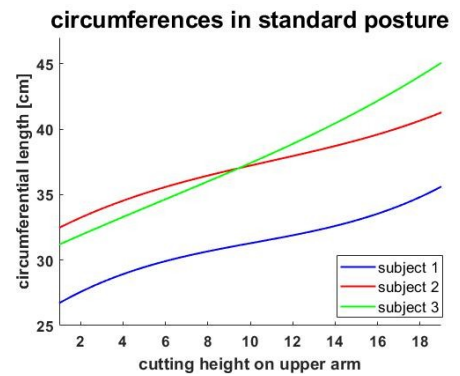

Fig. 6: Course of circumferences of the upper arm in standard posture for all subjects

Figure 7 shows the fitted average courses of circumferences in relaxed and tensed muscle condition. Despite the different circumferential lengths in standard posture between subject 1 and 3 , both subjects show similar courses and differences between tensed and relaxed conditions. The maximum difference for subject 1 is $0.9 \mathrm{~cm}$, for subject 3 it is $0.8 \mathrm{~cm}$ (compare Table 3).
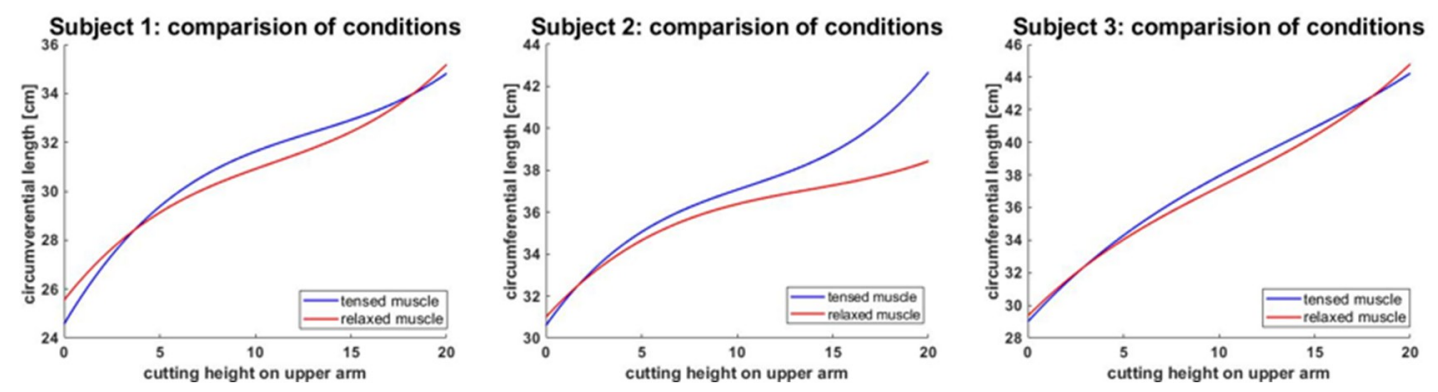

Fig. 7: Courses of circumferences of relaxed and tensed condition

Subject 2 presents the main differences in the higher parts of the upper arm, near the shoulder. The average difference is $1.1 \mathrm{~cm}$, but the maximum difference in circumference between the two conditions is $4.3 \mathrm{~cm}$.

Table 3: Circumferences in the relaxed and tensed condition [cm]

\begin{tabular}{|c|c|c|c|c|c|c|}
\hline & \multicolumn{2}{|c|}{ Subject 1 } & \multicolumn{2}{c|}{ Subject 2 } & \multicolumn{2}{c|}{ Subject 3 } \\
\hline Cutting height & relaxed & tensed & relaxed & tensed & relaxed & tensed \\
\hline $\mathbf{1}$ & 26.8 & 26.1 & 31.8 & 31.1 & 30.8 & 30.6 \\
\hline $\mathbf{2}$ & 27.2 & 26.8 & 32.9 & 32.8 & 31.4 & 31.3 \\
\hline $\mathbf{3}$ & 27.7 & 27.8 & 33.5 & 33.5 & 32.2 & 32.2 \\
\hline $\mathbf{4}$ & 28.4 & 28.3 & 34.1 & 34.2 & 33.1 & 33.0 \\
\hline $\mathbf{5}$ & 29.0 & 29.2 & 34.7 & 34.9 & 34.0 & 34.2 \\
\hline $\mathbf{6}$ & 29.6 & 29.9 & 35.1 & 35.5 & 34.7 & 35.0 \\
\hline $\mathbf{7}$ & 30.0 & 30.5 & 35.5 & 36.0 & 35.4 & 35.9 \\
\hline $\mathbf{8}$ & 30.4 & 31.1 & 35.8 & 36.5 & 36.1 & 36.7 \\
\hline $\mathbf{9}$ & 30.8 & 31.4 & 36.2 & 36.9 & 36.8 & 37.4 \\
\hline $\mathbf{1 0}$ & 31.5 & 32.3 & 37.0 & 37.7 & 37.7 & 38.5 \\
\hline $\mathbf{1 1}$ & 31.2 & 31.9 & 36.5 & 37.6 & 37.8 & 38.7 \\
\hline $\mathbf{1 2}$ & 31.3 & 32.1 & 36.6 & 37.6 & 38.4 & 39.2 \\
\hline $\mathbf{1 3}$ & 31.5 & 32.3 & 36.3 & 37.9 & 39.0 & 39.7 \\
\hline $\mathbf{1 4}$ & 31.8 & 32.4 & 36.7 & 38.2 & 39.7 & 40.2 \\
\hline $\mathbf{1 5}$ & 32.3 & 32.7 & 37.2 & 38.7 & 40.5 & 40.8 \\
\hline $\mathbf{1 6}$ & 32.9 & 33.1 & 37.9 & 39.3 & 41.4 & 41.3 \\
\hline $\mathbf{1 7}$ & 33.5 & 33.6 & 38.1 & 40.0 & 41.4 & 42.1 \\
\hline $\mathbf{1 8}$ & 33.8 & 33.8 & 38.5 & 41.0 & 42.5 & 42.3 \\
\hline $\mathbf{1 9}$ & 34.5 & 34.5 & 37.4 & 41.7 & 44.2 & 44.1 \\
\hline
\end{tabular}

Figure 8 shows the comparison of all subjects in mean circumferences and standard deviation for all conditions and postures. Subject 1 displays a small upper arm, where muscle circumferences vary within the conditions on an average of only $0.7 \mathrm{~cm}$. The maximum difference in muscle deformation is shown within the standard deviation of $1.3 \mathrm{~cm}$ for subject 1 . Although subject 3 has a much higher circumference of the upper arm than subject 1 , the deformation over postures and conditions does not vary a lot. His maximum deformation is $1.1 \mathrm{~cm}$, with an average deformation of $0.8 \mathrm{~cm}$ over the entire upper arm (Figure 8). 
Subject 1: Circumferences

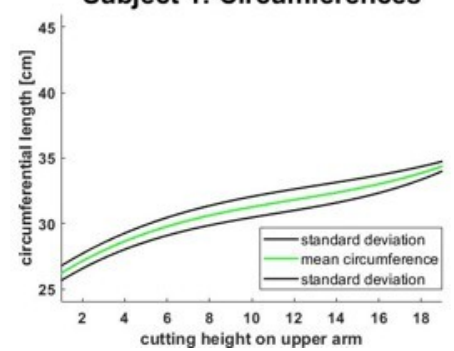

Subject 2: circumferences

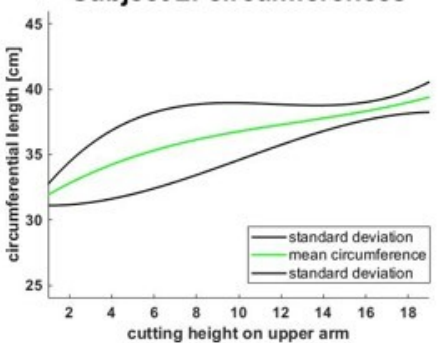

Subject 3: circumferences

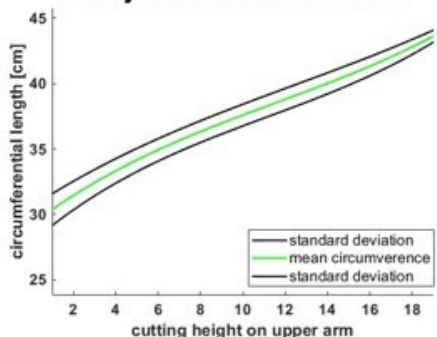

Fig. 8: Comparison of the mean circumferences \pm standard deviation for all subjects over all conditions and postures

In contrast, subject 2 shows a much higher variance of deformation in the lower part of the upper arm (figure 8), possibly resulting from a different fat to muscle ratio and a better-pronounced muscle belly. The average standard deviation for subject 2 is $1.7 \mathrm{~cm}$ with a maximum of $4.2 \mathrm{~cm}$ at the height of the physical marker, compare Table 4 (cutting height 10).

Table 4: Mean circumferences \pm standard deviation over all conditions and postures [mm]

\begin{tabular}{|c|c|c|c|}
\hline Cutting height & Subject 1 & Subject 2 & Subject 3 \\
\hline $\mathbf{1}$ & $26.6 \pm 0.7$ & $32.0 \pm 1.2$ & $30.7 \pm 1.1$ \\
\hline $\mathbf{2}$ & $27.0 \pm 0.6$ & $32.8 \pm 1.9$ & $31.4 \pm 1.0$ \\
\hline $\mathbf{3}$ & $27.8 \pm 0.6$ & $33.5 \pm 1.9$ & $32.2 \pm 1.1$ \\
\hline $\mathbf{4}$ & $28.3 \pm 0.5$ & $34.2 \pm 2.4$ & $33.0 \pm 1.1$ \\
\hline $\mathbf{5}$ & $29.1 \pm 0.5$ & $34.8 \pm 2.3$ & $34.1 \pm 1.1$ \\
\hline $\mathbf{6}$ & $29.7 \pm 0.6$ & $35.3 \pm 2.3$ & $34.9 \pm 1.1$ \\
\hline $\mathbf{7}$ & $30.3 \pm 0.6$ & $35.8 \pm 2.4$ & $35.7 \pm 1.0$ \\
\hline $\mathbf{8}$ & $30.8 \pm 0.6$ & $36.1 \pm 2.9$ & $36.4 \pm 0.5$ \\
\hline $\mathbf{9}$ & $31.1 \pm 1.2$ & $36.5 \pm 3.7$ & $37.1 \pm 0.4$ \\
\hline $\mathbf{1 0}$ & $31.9 \pm 1.3$ & $37.4 \pm 4.2$ & $38.1 \pm 0.3$ \\
\hline $\mathbf{1 1}$ & $31.5 \pm 0.8$ & $37.0 \pm 1.2$ & $38.3 \pm 1.1$ \\
\hline $\mathbf{1 2}$ & $31.7 \pm 0.8$ & $37.1 \pm 1.1$ & $38.8 \pm 1.0$ \\
\hline $\mathbf{1 3}$ & $31.9 \pm 0.7$ & $37.1 \pm 0.9$ & $39.3 \pm 1.0$ \\
\hline $\mathbf{1 4}$ & $32.1 \pm 0.7$ & $37.4 \pm 0.7$ & $39.9 \pm 1.0$ \\
\hline $\mathbf{1 5}$ & $32.5 \pm 0.6$ & $37.9 \pm 0.7$ & $40.6 \pm 0.9$ \\
\hline $\mathbf{1 6}$ & $33.0 \pm 0.6$ & $38.6 \pm 0.6$ & $41.4 \pm 0.8$ \\
\hline $\mathbf{1 7}$ & $33.6 \pm 0.5$ & $38.9 \pm 0.6$ & $41.8 \pm 0.6$ \\
\hline $\mathbf{1 8}$ & $33.8 \pm 0.5$ & $39.6 \pm 0.6$ & $42.4 \pm 0.4$ \\
\hline $\mathbf{1 9}$ & $34.5 \pm 0.6$ & $38.8 \pm 1.6$ & $44.2 \pm 0.5$ \\
\hline \multicolumn{4}{|c}{} \\
\hline \multicolumn{4}{|c|}{}
\end{tabular}

\subsection{Results of ellipse semi-major and semi-minor axis}

The more semi-major and semi-minor axis differ from one another, the more elliptical the upper arm is formed. In standard posture, subject 1 and 2 show similar arm shapes in relation to the elliptical forms, Subject 2 shows a distinctly elliptical shape in the lower part of the upper arm, whereas subject 1 presents an increasing elliptical form in the upper part of the segment, near the shoulder. Instead, subject 3 presents an upper arm that is quite round with only $\sim 7 \mathrm{~mm}$ difference between the semi-axes. In the upper segment, semi-major and semi-minor axes are changing, an implication, that the small elliptical shape changes its orientation. Figure 9 presents the fitted length of semi-minor and semi-major axes of the ellipses of all three participants in standard posture.
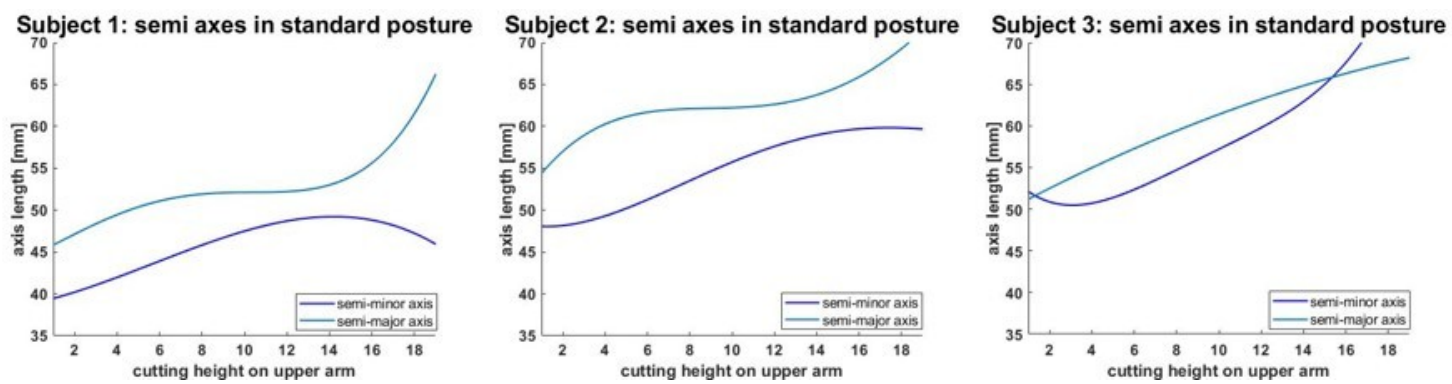

Fig.9: Length of ellipse axes in standard posture 
Table 5 presents the length values of the ellipse axes in standard posture for all cutting heights.

Table 5: Mean length of ellipse semi-axes in standard posture [ $\mathrm{mm}]$

\begin{tabular}{|c|c|c|c|c|c|c|}
\hline & \multicolumn{2}{|c|}{ Subject 1 } & \multicolumn{2}{c|}{ Subject 2 } & \multicolumn{2}{c|}{ Subject 3 } \\
\hline Cutting height & Semi-minor & Semi-major & Semi-minor & Semi-major & Semi-minor & Semi-major \\
\hline $\mathbf{1}$ & - & - & - & - & - & - \\
\hline $\mathbf{2}$ & 40.1 & 47.0 & 47.8 & 57.2 & 40.1 & 47.0 \\
\hline $\mathbf{3}$ & 41.1 & 48.3 & 48.8 & 58.5 & 41.1 & 48.3 \\
\hline $\mathbf{4}$ & 42.0 & 49.4 & 49.6 & 59.9 & 42.0 & 49.4 \\
\hline $\mathbf{5}$ & 43.0 & 50.4 & 50.4 & 61.3 & 43.0 & 50.4 \\
\hline $\mathbf{6}$ & 43.8 & 51.9 & 51.2 & 61.7 & 43.8 & 51.0 \\
\hline $\mathbf{7}$ & 44.6 & 51.4 & 51.9 & 62.2 & 44.6 & 51.4 \\
\hline $\mathbf{8}$ & 45.5 & 51.7 & 52.7 & 62.2 & 45.5 & 51.7 \\
\hline $\mathbf{9}$ & 46.5 & 52.0 & 53.8 & 62.2 & 46.5 & 52.0 \\
\hline $\mathbf{1 0}$ & 48.4 & 52.6 & 57.0 & 61.7 & 48.4 & 52.6 \\
\hline $\mathbf{1 1}$ & 48.0 & 51.9 & 57.3 & 62.3 & 48.0 & 51.9 \\
\hline $\mathbf{1 2}$ & 48.3 & 51.9 & 57.0 & 62.7 & 48.3 & 51.9 \\
\hline $\mathbf{1 3}$ & 48.9 & 52.5 & 58.5 & 62.8 & 48.9 & 52.5 \\
\hline $\mathbf{1 4}$ & 49.3 & 53.0 & 59.2 & 63.7 & 49.3 & 53.0 \\
\hline $\mathbf{1 5}$ & 64.9 & 66.1 & 59.0 & 65.0 & 64.9 & 66.1 \\
\hline $\mathbf{1 6}$ & - & - & 59.1 & 65.9 & - & - \\
\hline $\mathbf{1 7}$ & - & - & 60.2 & 67.2 & - & - \\
\hline $\mathbf{1 8}$ & - & - & - & - & - & - \\
\hline $\mathbf{1 9}$ & - & - & - & - & - & - \\
\hline
\end{tabular}

Figure 10 presents the fitted mean values of the axis length for semi-major and semi-minor axes in relaxed and tensed muscle condition.

The elliptical shape of subject 3's arm is now more apparent than in standard posture. Both axes raise their values from relaxed to tensed condition, but the shape itself does not change between the conditions.
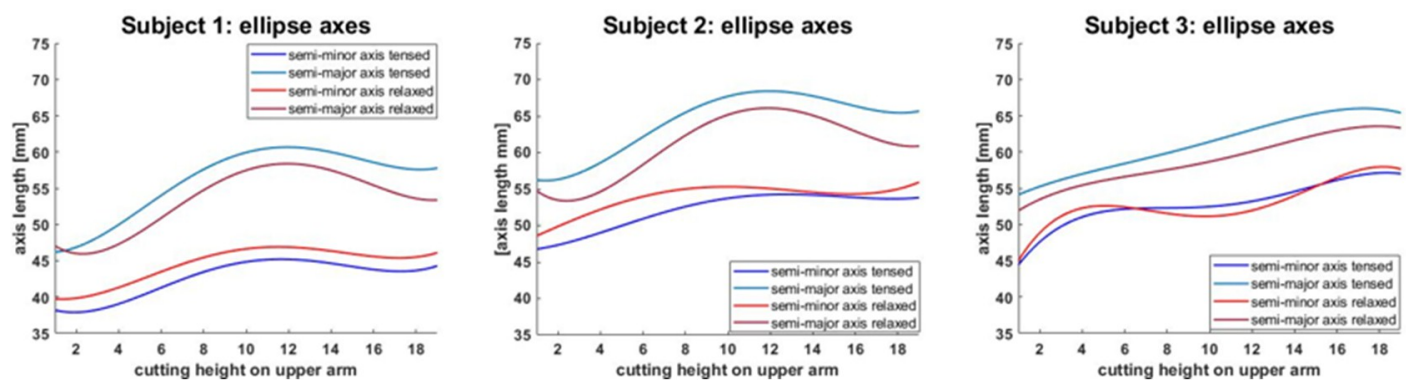

Fig. 10: Mean length of ellipse axes in two muscle conditions

Again subject 1 and 2, show similar changes between relaxed and tensed condition. The elliptical form of the upper arm becomes more distinct in the tensed condition, especially in the middle part of the upper arm, where the biceps has its center, compare cutting height 7-10 in Table 6.

Table 6: Mean length of ellipse semi-axes in relaxed and tensed muscle conditions [mm]

\begin{tabular}{|c|c|c|c|c|c|c|c|c|c|c|c|c|}
\hline & \multicolumn{4}{|c|}{ Subject 1} & \multicolumn{4}{|c|}{$\begin{array}{c}\text { Subject } 2 \\
\end{array}$} & \multicolumn{4}{|c|}{ Subject 3} \\
\hline & \multicolumn{2}{|c|}{ Semi-minor } & \multicolumn{2}{|c|}{ Semi-major } & \multicolumn{2}{|c|}{ Semi-minor } & \multicolumn{2}{|c|}{ Semi-major } & \multicolumn{2}{|c|}{ Semi-minor } & \multicolumn{2}{|c|}{ Semi-major } \\
\hline Cutting height & relaxed & tensed & relaxed & tensed & relaxed & tensed & relaxed & tensed & relaxed & tensed & relaxed & tensed \\
\hline 1 & 39.1 & 37.5 & 46.0 & 45.4 & 48.2 & 46.4 & 53.0 & 55.5 & 46.3 & 45.3 & 52.9 & 54.9 \\
\hline 2 & 40.1 & 38.1 & 46.2 & 47.2 & 50.2 & 47.4 & 54.3 & 56.6 & 48.8 & 46.9 & 53.4 & 55.1 \\
\hline 3 & 40.9 & 38.9 & 47.2 & 49.2 & 51.3 & 48.4 & 55.2 & 57.9 & 50.1 & 48.7 & 53.92 & 54.9 \\
\hline 4 & 41.7 & 39.5 & 48.4 & 50.2 & 52.3 & 49.4 & 56.1 & 59.2 & 48.4 & 50.1 & 52.62 & 55.9 \\
\hline 5 & 42.4 & 40.3 & 49.6 & 52.1 & 53.2 & 50.2 & 57.0 & 60.6 & 52.5 & 51.9 & 55.6 & 56.8 \\
\hline 6 & 43.3 & 41.0 & 50.6 & 53.6 & 53.9 & 50.8 & 57.7 & 61.8 & 53.4 & 52.7 & 57.0 & 58.6 \\
\hline 7 & 43.9 & 41.6 & 51.5 & 55.1 & 54.4 & 51.3 & 58.5 & 62.9 & 54.1 & 53.4 & 58.6 & 60.6 \\
\hline 8 & 44.4 & 42.3 & 52.1 & 56.1 & 54.7 & 51.8 & 59.2 & 63.9 & 54.8 & 54.1 & 59.9 & 62.4 \\
\hline 9 & 45.1 & 42.7 & 52.8 & 56.6 & 55.1 & 52.2 & 60.0 & 64.7 & 55.8 & 54.9 & 61.0 & 64.0 \\
\hline 10 & 48.3 & 47.2 & 61.7 & 63.2 & 53.9 & 54.2 & 67.7 & 70.2 & 48.1 & 46.9 & 59.3 & 57.0 \\
\hline 11 & 47.4 & 46.2 & 60.3 & 61.9 & 56.5 & 54.8 & 70.0 & 70.5 & 45.9 & 50.0 & 55.1 & 58.9 \\
\hline 12 & 46.7 & 45.2 & 59.0 & 60.9 & 55.7 & 54.4 & 68.1 & 68.8 & 47.4 & 53.6 & 55.5 & 63.1 \\
\hline 13 & 46.3 & 44.6 & 57.6 & 59.9 & 55.4 & 54.8 & 66.5 & 68.0 & 53.7 & 55.4 & 61.1 & 64.2 \\
\hline 14 & 47.2 & 44.4 & 57.6 & 59.2 & 54.1 & 54.2 & 64.1 & 66.7 & 57.4 & 56.5 & 63.7 & 65.7 \\
\hline 15 & 45.4 & 43.9 & 55.5 & 58.7 & 54.0 & 53.7 & 62.5 & 65.7 & 56.4 & 55.3 & 62.6 & 65.3 \\
\hline 16 & 45.2 & 43.7 & 54.7 & 58.4 & 53.9 & 53.6 & 61.4 & 66.5 & 57.0 & 56.4 & 63.3 & 66.3 \\
\hline 17 & 45.1 & 43.5 & 54.1 & 58.1 & 54.6 & 53.3 & 61.7 & 66.0 & 57.5 & 55.4 & 64.5 & 65.6 \\
\hline 18 & 45.3 & 43.3 & 53.6 & 57.6 & 54.8 & 53.4 & 61.1 & 65.9 & 56.8 & 56.6 & 63.5 & 66.2 \\
\hline 19 & 46.4 & 44.6 & 53.8 & 57.8 & 56.1 & 54.2 & 61.5 & 65.4 & 57.6 & 57.5 & 62.4 & 64.9 \\
\hline
\end{tabular}

Figure 11 presents the mean \pm standard deviation length of semi-major and semi-minor axes over all conditions and postures captured for all three subjects separately. Subject 1 presents a relatively constant shape in relation to the elliptical shape, whose elliptical axes differ only one to three 
millimeters over all conditions and postures. Subject 2 shows a course of axis-lengths over the arm segment, which is similar to subject 1 , with elevated values and a slightly increased variation of standard deviations. In contrast, the elliptical form of the upper arm varies the most in subject 3 . The inconsistent behavior is represented by the interception of the standard deviation with the mean values of the other semi axis.
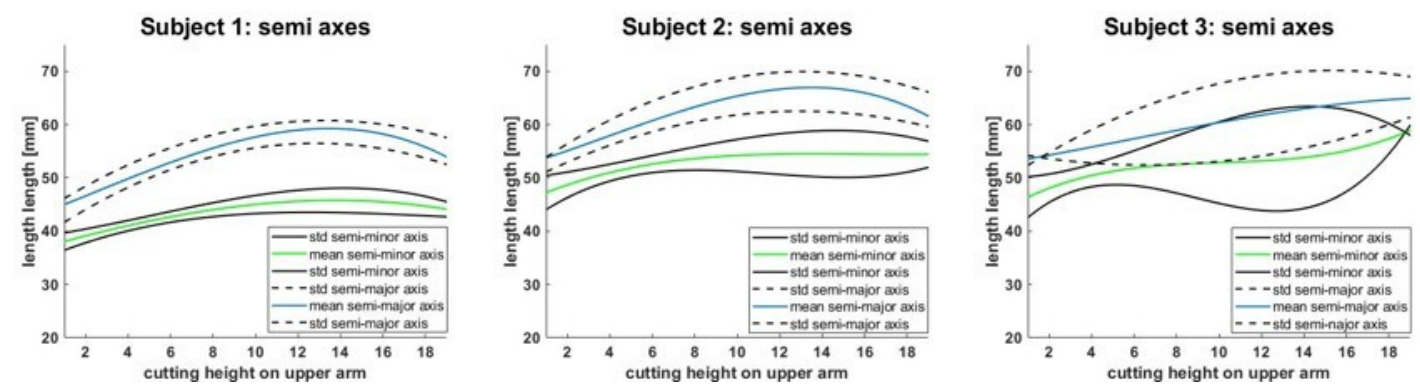

Fig. 1: Mean length of ellipse axes over all postures and conditions

Table 7 presents the mean values \pm standard deviation of ellipse axes over all postures and muscle conditions for the three participants.

Table 7: Mean \pm standard deviation length of ellipse semi-axes over all postures and conditions over all postures and conditions [mm]

\begin{tabular}{|c|c|c|c|c|c|c|}
\hline & \multicolumn{2}{|c|}{ Subject 1 } & \multicolumn{2}{c|}{ Subject 2 } & \multicolumn{2}{c|}{ Subject 3 } \\
\hline Cutting height & Semi-minor & Semi-major & Semi-minor & Semi-major & Semi-minor & Semi-major \\
\hline $\mathbf{1}$ & $38,6 \pm 1,0$ & $45,8 \pm 2,2$ & $47,4 \pm 3,7$ & $54,2 \pm 2,7$ & $45,8 \pm 3,5$ & $53,9 \pm 2,5$ \\
\hline $\mathbf{2}$ & $39,3 \pm 1,3$ & $46,7 \pm 1,8$ & $48,8 \pm 1,6$ & $55,5 \pm 2,0$ & $47,8 \pm 2,0$ & $54,3 \pm 2,4$ \\
\hline $\mathbf{3}$ & $40,0 \pm 1,4$ & $48,2 \pm 1,8$ & $49,9 \pm 1,8$ & $56,6 \pm 1,7$ & $49,4 \pm 2,0$ & $54,5 \pm 2,1$ \\
\hline $\mathbf{4}$ & $40,6 \pm 1,5$ & $49,4 \pm 2,0$ & $50,9 \pm 1,8$ & $57,7 \pm 1,7$ & $49,2 \pm 6,1$ & $54,3 \pm 3,5$ \\
\hline $\mathbf{5}$ & $41,4 \pm 1,5$ & $50,9 \pm 2,3$ & $51,7 \pm 1,7$ & $58,9 \pm 1,9$ & $52,2 \pm 2,3$ & $56,3 \pm 1,2$ \\
\hline $\mathbf{6}$ & $42,2 \pm 1,3$ & $52,2 \pm 2,6$ & $52,4 \pm 1,7$ & $59,8 \pm 2,0$ & $53,1 \pm 2,5$ & $57,8 \pm 1,4$ \\
\hline $\mathbf{7}$ & $42,8 \pm 1,3$ & $53,3 \pm 2,8$ & $52,9 \pm 1,8$ & $60,7 \pm 2,3$ & $53,8 \pm 2,7$ & $59,6 \pm 1,7$ \\
\hline $\mathbf{8}$ & $43,4 \pm 1,2$ & $54,2 \pm 3,0$ & $53,3 \pm 1,8$ & $61,6 \pm 2,7$ & $54,4 \pm 2,5$ & $61,2 \pm 1,9$ \\
\hline $\mathbf{9}$ & $43,9 \pm 1,2$ & $54,8 \pm 2,9$ & $53,7 \pm 1,7$ & $62,4 \pm 2,8$ & $55,3 \pm 2,4$ & $62,6 \pm 2,2$ \\
\hline $\mathbf{1 0}$ & $47,8 \pm 1,0$ & $62,5 \pm 1,0$ & $54,1 \pm 5,4$ & $69,0 \pm 5,9$ & $47,5 \pm 10,8$ & $58,1 \pm 12,3$ \\
\hline $\mathbf{1 1}$ & $46,8 \pm 0,8$ & $61,2 \pm 1,0$ & $55,6 \pm 3,3$ & $70,3 \pm 3,9$ & $48,0 \pm 10,7$ & $57,2 \pm 11,8$ \\
\hline $\mathbf{1 2}$ & $45,9 \pm 0,9$ & $60,1 \pm 1,2$ & $55, \pm 13,5$ & $68,5 \pm 3,7$ & $50,5 \pm 11,5$ & $59,3 \pm 11,5$ \\
\hline $\mathbf{1 3}$ & $45,5 \pm 1,7$ & $58,9 \pm 1,3$ & $55,1 \pm 2,6$ & $67,3 \pm 3,9$ & $54,6 \pm 12,0$ & $62,7 \pm 11,6$ \\
\hline $\mathbf{1 4}$ & $45,8 \pm 6,1$ & $58,4 \pm 3,3$ & $54,2 \pm 4,4$ & $65,4 \pm 4,1$ & $56,9 \pm 9,5$ & $64,8 \pm 8,1$ \\
\hline $\mathbf{1 5}$ & $44,7 \pm 2,1$ & $57,1 \pm 2,0$ & $53,9 \pm 4,5$ & $64,2 \pm 4,0$ & $55,9 \pm 8,8$ & $64,0 \pm 7,8$ \\
\hline $\mathbf{1 6}$ & $44,5 \pm 2,1$ & $56,6 \pm 2,3$ & $53,8 \pm 4,5$ & $64,0 \pm 4,1$ & $56,7 \pm 6,0$ & $64,9 \pm 6,5$ \\
\hline $\mathbf{1 7}$ & $44,4 \pm 1,8$ & $56,2 \pm 2,5$ & $54,0 \pm 4,1$ & $63,9 \pm 3,1$ & $56,4 \pm 2,0$ & $65,1 \pm 3,4$ \\
\hline $\mathbf{1 8}$ & $44,4 \pm 1,6$ & $55,6 \pm 2,7$ & $54,1 \pm 4,0$ & $63,6 \pm 3,1$ & $56,7 \pm 2,2$ & $64,9 \pm 3,0$ \\
\hline $\mathbf{1 9}$ & $45,5 \pm 1,2$ & $55,8 \pm 2,5$ & $55,2 \pm 1,8$ & $63,5 \pm 2,8$ & $57,5 \pm 1,8$ & $63,7 \pm 2,5$ \\
\hline
\end{tabular}

\section{Discussion}

Without adequate anthropometric data and knowledge of its changes in specific segment postures and motion, the fitting, force transfer of human-machine-interface and safety issues may not sufficient. At best, concerning force transfer and fitting the designing of human-machine-interface is based on anthropometric data studies that are tailored to the specific body segments, which are to be connected with the exoskeleton and the postures and movements that are to be supported by the exoskeleton. The results of the pilot scanning study reveal a strong indication that individual human-machine-interfaces are needed to achieve satisfaction concerning fitting, force transfer and safety aspects.

This paper presented an approach that considers eleven different postures, with different degrees in arm flexion, abduction, pronation and supination as well as two different muscle conditions that represents usual working postures from assembly, production and manufacturing. In order to minimize the expenditure of the acquisition study the number of postures might be reduced to those containing the most information. To realize such a reduction further examination of the results is required. In this context it should be considered that any reduction of the observed postures will decrease the information contained in the variances and might ignore substantial behavior of the soft tissue. To achieve a trade-off, weighting of the postures is required. This depends on a more precise analysis of the movements supported by the exoskeleton and exposures from the work environment (e.g., duration of the adopted postures adopted).

Beyond the acquisition of the 3D-data, this paper introduced a feasible option for reducing the shape data into geometric patterns. This allows the usage of the data for an automated design of the individual human-machine-interface. Automating this currently labor-intensive design task, enables a 
quick personalization of the predesigned human-machine-interface, paving the way of individualized exoskeletons from a modular system to the mass market.

The individual form and the resilient materials allow the soft tissue to move within the human-machine interface and ensure safety requirements of the human-machine-interface in exoskeletons are met. This includes the protection of the sensitive vascular and neuronal structures in the soft tissue, especially the medial bicipital groove.

For the pilot study, subjects were chosen according to their body type in order to integrate different arm shapes to verify the feasibility of the study and determine the usability of the emerging results for individual human-machine-interfaces. The subjects not only showed a difference in circumferences but also in the increasing rate of their conical shapes from elbow to shoulder, a difference in its behavior between tensed and relaxed muscle conditions and the degree and direction of deformation of the soft tissue.

The results require further verification in a large-scale study to evaluate the influence of the factors revealed in this pilot study. Some factors to be mentioned in this context are the dependence of the mean circumference itself, the dependence of the predisposition of the musculature and its physical condition, the influence of the subcutaneous fat layer and its behavior against gravity.

\section{Ethical Statement}

This research was approved by the Board for Ethical Issues at Innsbruck University (No.36/2019) on $9^{\text {th }}$ August 2019.

\section{References}

[1] M. P. de Looze, T. Bosch, F. Krause, K. S. Stadler, \& L. W. O'Sullivan, Exoskeletons for industrial application and their potential effects on physical workload, Ergonomics, Vol. 59, No. 5, 2015, pp.671-681. https://doi.org/10.1080/00140139.2015.1081988.

[2] L. Levesque, S. Pardoel, M. Doumit, \& Z. Lovrenovic, Experimental comfort assessment of an active exoskeleton interface, in IEEE International Symposium on Robotics and Intelligent Sensors (IRIS) 2017 Ottawa Canada, 2017, pp. 5-7. https://doi.org/10.1109/IRIS.2017.8250095.

[3] C. Linnenberg, J. Klabunde, R. S. Weidner, \& J. P. Wulfsberg, Entwicklungsansatz für physische Mensch-Technik-Schnittstellen von Exoskeletten, in 3. transdisziplinäre Konferenz „Technische Unterstützungssysteme, die die Menschen wirklich wollen" am 11. und 12. Dezember 2018, 2018, pp. 117-126.

[4] ISO 8559-2:2017 (E) Size designation of Cloth - Part 2: Primary and secondary dimension indicators, 2017, pp. 1-37.

[5] Specialty Material Handling Products Operation General Electric Company, Final Report on Hardiman I Prototype for Machine Augmentation of Human Strength and Endurance, 1971.

[6] T. Bosch, J. van Eck, K. Knitel, \& M. de Looze, The effects of a passive exoskeleton on muscle activity, discomfort and endurance time in forward bending work, Appl. Ergon., Vol. 54, 2016, pp. 212-217. https://doi.org/10.1016/j.apergo.2015.12.003.

[7] F. Schröter, R. Weidner, P. Dehmel, T. Jakobsen, \& J. Wulfsberg, Der beflügelte Mensch gesteigerte Konzentration durch Unterstützungssysteme in der Produktion, in 51. DGPs-Kongress Frankfurt am Main.

[8] M. B. Yandell, B. T. Quinlivan, D. Popov, C. Walsh, \& K. E. Zelik, Physical interface dynamics alter how robotic exosuits augment human movement: implications for optimizing wearable assistive devices, J. Neuroeng. Rehabil., Vol. 14, No. 1, 2017, pp.1-11. https://dx.doi.org/10.1186\%2Fs12984-017-0247-9.

[9] Y. He, D. Eguren, T. P. Luu, \& J. L. Contreras-Vidal, Risk management and regulations for lower limb medical exoskeletons: A review, Med. Devices Evid. Res., Vol. 10, 2017, pp. 89-107. https://dx.doi.org/10.2147\%2FMDER.S107134.

[10] C. Plegge, Bedeutung und kinematische Untersuchung der Passform eines aktiven Exoskelettes für die untere Extremität, in Band zur zweiten Transdisziplinären Konferenz „Technische Unterstützungssysteme, die die Menschen wirklich wollen“, 2016.

[11] S. Habetha \& U. Golbach, Sichtung, Sammlung und Auswertung der verfügbaren Belege zur Wirksamkeit von Bandagen und Orthesen am Knie. IGSF Institut für Gesundheits-System-Forschung GmbH, Kiel, 2014. 
[12] J. V Durá, G. Caprara, M. Cavallaro, A. Ballarino, C. Kaiser, \& D. Stellmach, New technologies for the flexible and eco-efficient production of customized products for people with special necessities: Results of the FASHION-ABLE project, 2014 International Conference on Engineering, Technology and Innovation (ICE)., 2017, pp. 1-7. https://doi.org/10.1109/ICE.2014.6871558.

[13] C. Kaiser, A. Artschwager, D. Stellmach, T. Schmeltzpfenning, J. V. Dura, \& M. Stohr, Development of a made to measure process for customizing lumbar orthotics for obese people, 2014 Int. Conf. Eng. Technol. Innov. Eng. Responsible Innov. Prod. Serv. ICE 2014, November, 2014. https://doi.org/10.1109/ICE.2014.6871602.

[14] A. Seidl, R. Trieb, \& H.-J. Wirsching, SizeGermany - die neue Deutsche Reihenmessung Konzeption, Durchführung und erste Ergebnisse, in Produkt- und Produktions-Ergonomie Aufgabe für Entwickler und Planer, Gesellschaft für Arbeitswissenschaft, Ed. Dortmund: GfA-Press, 2008, pp. 391-394.

[15] A. Seidl, R. Trieb, H.-J. Wirsching, A. Smythe, \& T. Guenzel, SizeNorthAmerica---The New North American Anthropometric Survey: Conceptual Design, Implementation and Results, in Advances in Physical Ergonomics and Human Factors, 2016, pp. 457-468. https://doi.org/10.1007/978-3-319-41694-6_46.

[16] Assyst GmbH, Vidya $3 D \quad$ mit Realitätsplus, 2018. https://www.human-solutions.com/fashion/front_content.php?idcat=150\&lang=5. accessed: 2017.

[17] P. H. Bubb, Automobilergonomie. 2015.

[18] S. Morlock, C. Lörcher, \& A. Schenk, Entwicklung eines ergonomisch- und bewegungsorientierten Größensystems für Funktionsmaße zur optimierten Gestaltung von Berufs- und Schutzbekleidung. Schlussbericht zum IGF-Vorhaben 18993 N, Bönnigkeim, 2018.

[19] O. Gal, fit_ellipse. MATLAB Central File Exchange, https://de.mathworks.com/matlabcentral/fileexchange/3215-fit_ellipse, accessed: 2018.

[20] S. Morlock, A. Schenk, \& M. Harnisch, Schlussbericht zum IGF-Vorhaben: Passformgerechte und Bekleidungsphysiologisch optimierte Bekleidungskonstruktion für Männer mit großen Größen unterschiedlicher Körpermorphologien. Hohenstein Institute, 2015. 